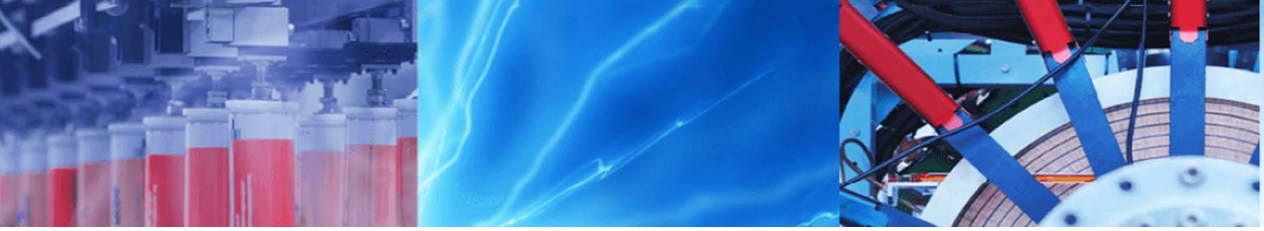

Research Article

\title{
Preparation of porous graphene/carbon nanotube composite and adsorption mechanism of methylene blue
}

\author{
Yangfan Huang ${ }^{1} \cdot$ Jiameng Zhu ${ }^{1} \cdot$ Huie Liu ${ }^{1} \cdot$ Zhenyou Wang $^{1} \cdot$ Xiuxia Zhang ${ }^{1}$
}

(c) Springer Nature Switzerland AG 2018

\begin{abstract}
The graphene oxide (GO) and modified carbon nanotubes (MCNTs) were dispersed in water and mixed with toluene to form homogeneous emulsion, then the porous graphene-carbon nanotubes composites (MCG) were prepared through hydrothermal reaction. The adsorption mechanism of MCG was investigated by adsorption methylene blue. The morphology of MCG was analyzed by scanning electron microscope and transmission electron microscopy. It was found that MCG had rich micropore structures and MCNTs were interlaced on graphene sheets. The pore size of MCG can be effectively controlled by changing the volume ratio of toluene to the GO/MCNTs solution in the emulsion. MCG was characterized by X-ray diffractometer, Raman spectrometer, $\mathrm{X}$ ray photoelectric instrument and other analytical instruments. It was found that MCG had more oxygen functional groups. The experimental results show that kinetics can be well-described by pseudo-second-order model. The smaller the pore size, the higher the equilibrium adsorption capacity and the slower the adsorption rate. The adsorption thermodynamic parameters show that the adsorption process is spontaneous and belongs to physical adsorption, and high temperature is beneficial to adsorption. The fitting results of MCG-5 equilibrium adsorption data are matched with the Langmuir model and the saturated adsorption capacity is $232 \mathrm{mg} \mathrm{g}^{-1}$. After five cycles of adsorption-desorption operation, the adsorption capacity of MCG-5 decreases slightly.
\end{abstract}

Keywords Graphene $\cdot$ Carbon nanotubes $\cdot$ Methylene blue $\cdot$ Thermodynamics $\cdot$ Kinetics

Mathematics Subject Classification 92E99

JEL Classification Q53

\section{Introduction}

The development of papermaking, printing and dyeing and textile industry has caused a large amount of water pollution, among which dye wastewater has the characteristics of large toxicity, difficult treatment and strong stability [1,2]. At present, the common treatment methods of dye wastewater include adsorption, photocatalytic degradation, chemical oxidation and membrane separation
[3-8]. These methods can solve the problem of dye wastewater to a certain extent, but at the same time, there are also shortcomings such as harsh operating conditions, secondary pollution, low efficiency and high cost. Adsorption is also an important water treatment method, while finding an efficient adsorbent is the key for its application [9].

Methylene blue (MB) is an important organic dye widely used in textile, dyeing, printing, pesticide, and coating for paper stock [10]. Because of its aromatic ring, MB is highly

$\triangle$ Huie Liu, liuhuie@upc.edu.cn; Yangfan Huang, hyf_upc@163.com; Jiameng Zhu, zjm13061348809@163.com; Zhenyou Wang, 15314221338@163.com; Xiuxia Zhang, zhxiuxia@upc.edu.cn | 'State Key Laboratory of Heavy Oil Processing, College of Chemical Engineering, China University of Petroleum (East China), West Changjiang Road 66\#, Economic and Technological Development Zone, Qingdao City 266580, Shandong, People's Republic of China. 
toxic and very difficult to degrade $[11,12]$. Consequently, MB must be removed from wastewater before discharging.

Two dimensional graphene and graphite oxide $(\mathrm{GO})$ are flaky nanomaterials. Their large surface area, rich adsorption site and high mechanical strength also make them become excellent adsorption materials [13-15]. However, there will be difficult to recycle the powder materials, and it can cause secondary pollution. In order to solve the problem, assembling two-dimensional materials into three-dimensional materials have been regarded. Ma et al. [16] prepared graphite oxide aerogels (GO-SA) and graphene aerogels (RGO-GA) from $\mathrm{GO}$ and two-dimensional graphene, respectively. It was found that the mechanism of methylene blue (MB) adsorption was different. The negative charge of oxygen containing functional groups on the GO-SA surface were favorable for the adsorption of cationic MB. RGO-SA had aromatic ring structure, and MB molecule produced $\pi-\pi$ conjugation with RGO-SA. So, the functional groups on aerogels can influence the adsorption mechanism for $\mathrm{MB}$ and modification of the material using different functional groups can be carried out according to need.

Carbon nanotube (CNT) is easily oxidized and modified, and more oxidation functional groups can be formed on the surface to enhance the adsorption capacity [17]. And its larger specific surface area is beneficial to adsorption. Ai et al. [18] loaded the nano Fe particles on the oxidized multi walled carbon nanotubes (M-MWCNTs), and found that M-MWCNTs has a strong adsorption capacity to methylene blue, reaching $48.06 \mathrm{mg} \mathrm{g}^{-1}$. At the same time, Single-wall carbon nanotubes (CNTs) possess strong tensile strength [19] and elastic modulus [20] since the buckling of the $s p^{2}$-hybridized bonds [21]. It may increase the stability and ductility of $3 \mathrm{D}$ graphene-based macrostructures by blending CNTs with GO since the flexibility of GO caused by its $s p^{2}$-hybridized bonds will be reduced $[22,23]$.

Generally, both CNTs and graphene have good toughness and high strength, but neat graphene and CNT aerogels have weak elasticity. It has been reported that $\mathrm{GO}$ and CNTs may be mixed to form composite or aerogels [24, 25]. Recently, the combination of CNTs and graphene to prepare 3D composite aerogels for adsorption has aroused great attention. For example, it reported a "sol-cryo" method for the synthesis of carbon aerogels with a density of $0.16 \mathrm{mg} \mathrm{cm}^{-3}$ and these aerogels exhibited excellent mechanical properties [26]. Also, there are many methods to prepare the graphene aerogels: chemical vapor deposition, hydrothermal reaction, sol-gel method, soft template method and so on.

The oxygen-containing groups of $\mathrm{GO}$ are mainly on the edge of lamellar, and the middle is polymerized aromatic ring structure, so it has hydrophilicity and lipophilicity. These two affinity can make GO behave as a molecular and colloidal stabilizer. Therefore, it provides advantage for the preparation of aerogels by using soft template method. On the one hand, as a molecular stabilizer, GO can make the carbon nanotubes stabilized in water. On the other hand, When GO is used as a colloidal stabilizer, the oil in water $(\mathrm{O} / \mathrm{W})$ type emulsion $[27,28]$ can be prepared.

In this paper, GO was used as stabilizer, and the modified multi walled carbon nanotubes were dispersed in the solution. After adding the aromatic oil phase, toluene, a stable O/W emulsion was formed. Porous graphene/modified carbon nanotubes 3D composites (MCG) were prepared after high temperature hydrothermal reaction. As a $3 \mathrm{D}$ porous material, $\mathrm{MCG}$ is a promising adsorbent for $\mathrm{MB}$. The adsorption properties of $M B$ and their dependence on a variety of parameters as well as adsorption isotherm, kinetic, and thermodynamic characteristics were determined for MCG.

\section{Experimental}

\subsection{Materials}

Flake graphite powder and sodium nitrate $\left(\mathrm{NaNO}_{3}\right)$ were purchased from Aladdin's Reagent Company. Multi-wall carbon nanotubes (CNTs) was provided by the Tsinghua University. Potassium ermanganate $\left(\mathrm{KMnO}_{4}\right), 98 \mathrm{wt} \%$ sulfuric acid $\left(\mathrm{H}_{2} \mathrm{SO}_{4}\right), 68 \mathrm{wt} \%$ nitric acid $\left(\mathrm{HNO}_{3}\right), 36 \mathrm{wt} \%$ hydrochloric acid $(\mathrm{HCl}), 30 \mathrm{wt} \%$ hydrogen peroxide $\left(\mathrm{H}_{2} \mathrm{O}_{2}\right)$, L-ascorbic acid (L-AA), acetone, toluene and methylene blue were purchased from the chemical reagent company of national pharmaceutical chemical group. The deionized water was provided by heavy oil Laboratory of China University of Petroleum (East China).

\subsection{Methods}

\subsubsection{Preparation of graphite oxide}

Graphite Oxide (GO) was prepared through modified Hummers method $[29,30] .0 .5 \mathrm{~g}$ flake graphite powder and $\mathrm{NaNO}_{3}$ are added into $23 \mathrm{~mL} \mathrm{H} \mathrm{SO}_{4}(98 \mathrm{wt} \%)$. The mixture was stirred under $10^{\circ} \mathrm{C}$ and then $3 \mathrm{~g} \mathrm{KMnO}_{4}$ was added to the mixture slowly. The temperature was increased to $35^{\circ} \mathrm{C}$ and the stirring continued for $1 \mathrm{~h}$, and thus a uniform thick pulp formed. The pulp was warmed up to $95^{\circ} \mathrm{C}$ thereafter, $40 \mathrm{~mL}$ deionized water was added and stirring was kept for another $0.5 \mathrm{~h}$. Then the solution was moved out, and $100 \mathrm{~mL}$ deionized water and $3 \mathrm{~mL} \mathrm{H}_{2} \mathrm{O}_{2}$ were added into the solution. At this time, the color of the solution is yellow. After being washed to neutral, GO powder can be obtained after being freeze-dried in the lyophilizer (FD$1 \mathrm{~A}-50$ ) for $48 \mathrm{~h}$. 


\subsubsection{Modification of carbon nanotubes}

The carbon nanotubes (CNTs) was modified as follows [31]: CNTs was added into $\mathrm{HNO}_{3}$, keeping the temperature of reaction at $75^{\circ} \mathrm{C}$ for $11 \mathrm{~h}$. After filtration, the powder was washed to neutral. Then the modified carbon nanotubes (MCNTs) was obtained through freeze-drying in the lyophilizer (FD-1A-50) for $48 \mathrm{~h}$.

\subsubsection{Preparation of graphene/carbon nanotube 3D composites}

A certain amount of $\mathrm{GO}$ was dissolved in deionized water. $5 \mathrm{mg} \mathrm{mL}^{-1} \mathrm{GO}$ solution was prepared through ultrasonic dispersion for $1 \mathrm{~h}$. MCNTs was then added into the GO solution with ultrasonic dispersion continued for another $1 \mathrm{~h}$. The mass ratio between $\mathrm{GO}$ and MCNTs (G/C) was 2:1. A certain amount of L-AA was added and the $\mathrm{pH}$ value of the solution was adjusted to 2 by $1 \mathrm{~mol} \mathrm{~L}^{-1} \mathrm{HCl}$. And then a certain volume of toluene was added into the above solution. Homogeneous emulsion [32] formed after being stirred for $3 \mathrm{~min}$ at $15,000 \mathrm{r} \mathrm{min}^{-1}$ by digital display high speed homogenizer (FJ200-S), which then was put in Teflon high-pressure autoclave under $95^{\circ} \mathrm{C}$ for $8 \mathrm{~h}$ in oven (FCD-3000) and hydrogel was obtained. The hydrogels were washed repeatedly with acetone and deionized water. Graphene/carbon nanotube aerogels (MCG) were obtained through being freeze-dried in the lyophilizer (FD-1A-50) for $48 \mathrm{~h}$. The volume of toluene was adjusted, and the volume ratio between toluene and the GO/MCNTs solution (T/S ratio) was controlled at 2:10, 5:10 and 8:10 and MCG-2, MCG-5 and MCG-8, respectively, were named as abbreviation for convenience.

A schematic diagram of the preparation of MCG is shown in Fig. 1.

\subsubsection{Adsorption of MB by MCG}

The experimental method of methylene blue (MB) adsorption is as follows: a piece of MCG was placed in a flask with $200 \mathrm{~mL}$ MB solution. The solution was magnetically stirred at $298 \mathrm{~K}$. Samples were taken at a certain time interval and its absorbance was measured with ultraviolet spectrophotometer. The type of MCG and operation temperature were varied to explore the influence of MCG type and temperature on adsorption effects of MCG on MB. The concentration of MB solution can be obtained by Lambert-Beer law. The adsorption amount of $q_{t}$ at the instant $t$ can be calculated using Eq. (1).

$q_{t}=\frac{\left(C_{0}-C_{t}\right) V}{m}$
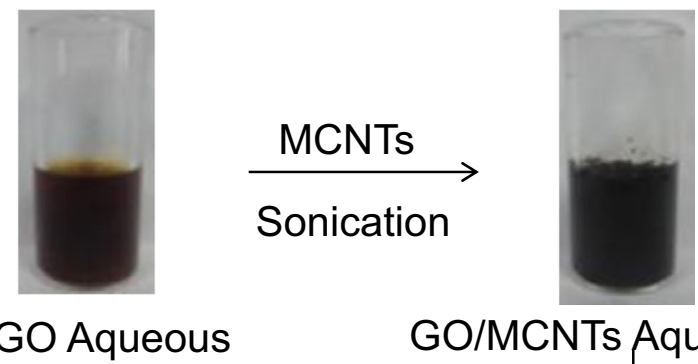

GO Aqueous

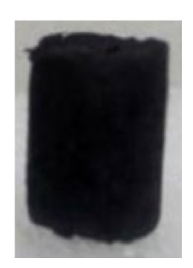

GO/MCNTs Aqueous $\downarrow \mathrm{C}_{7} \mathrm{H}_{8}$

MCG
Hydrothermal

\section{Freeze Dried}

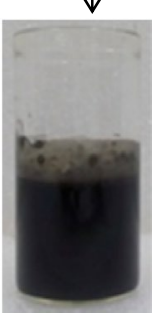

Emulsion

Fig. 1 Schematic diagram of the preparation of MCG

where $q_{t}$ is the adsorption amount of MB at $t$ moment, $\mathrm{mg} \mathrm{g}^{-1} ; C_{0}$ and $C_{\mathrm{t}}$ is the concentration of MB solution at the initial time and $t$ moment respectively, $\mathrm{mg} \mathrm{L}^{-1} ; V$ is the volume of the solution, $L ; m$ is the mass of MCG, $g$.

The desorption and cyclic adsorption processes are as follows [33]: MCG-5 reaching adsorption equilibrium at $298 \mathrm{~K}$ was put into ethanol to wash out the adsorbed MB. After washing several times, the ethanol became colorless, and then MCG-5 was freeze-dried in the lyophilizer (FD-1 A-50) for $48 \mathrm{~h}$ and then used to adsorb MB at $298 \mathrm{~K}$ again, which was repeated for 5 times.

\subsubsection{Characterization}

The droplet micromorphology of the emulsion was observed by the Japanese Olympus CX31 optical microscope (OM). The morphology of the MCG samples was analyzed by the German Zeiss Gemini 500 scanning electron microscope (SEM) and the Japanese JEM-2010 transmission electron microscope (TEM). The infrared spectrum was measured by IS10 Thermo Scientific full reflection Fourier transform infrared spectrometer. The Raman spectrum was measured by the DXR Microscope Raman spectrometer. The X-ray diffraction spectrum (XRD) was measured by the X'pert PROMPD $X$ ray diffractometer in Holland. The X-ray photoenergy spectra (XPS) was measured by Escalab 250XI photoelectron. The concentration of MB was measured by the UV-752 ultraviolet spectrophotometer from Shanghai RuoKe co, under the absorption wavelength of $664 \mathrm{~nm}$. 


\section{Results and discussion}

\subsection{Characterization of MCG}

Figure 2 gives the SEM images of MCGs, TEM image of MCG-5 and the optical microscopy image of the emulsion under T/S ratio of 10:5. The optical microscopy image of the emulsion in Fig. $2 \mathrm{f}$ shows that the droplets are spherical in shape and the diameter is about 10-30 $\mu \mathrm{m}$.

Figure 2a-c are the SEM images of MCG-2, MCG-5 and MCG-8. It can be seen that the MCGs have rich micropores, and with the increase of T/S ratio, the pore size of MCG becomes smaller and the microstructure is more abundant. The stacking between graphene layers for MCG-2 is less significant than that of MCG-8, which indicates that the effect of the soft template, toluene droplets, in MCG forming is weakened with the increase of T/S ratio, and some pores were damaged and pores with smaller size was formed. Figure $2 d$ is a SEM image at high magnification of MCG-5. It shows that many wrinkles exist on the surface of graphene layers in MCG-5, and MCNTs overlaps with each other on the surface of graphene. This structure enhances the roughness of the surface and generates a large number of mesoporous channels. These channels increase the specific surface area and pore volume of MCG, which is beneficial to improve the adsorption capacity of MCG. The TEM of MCG-5 (Fig. 2e) can clearly show the lamellar structure of graphene and tubular structures of MCNTs. MCNTs are interlaced on graphene lamellar, indicating that GO plays an important role in dispersing MCNTs. At the same time, MCNTs enhance the stability of micro-pore structure and reduce the stacking [34] of graphene lamellar. So, the MCG-5 was used as main adsorbent for MB in later adsorption experiments.

Figure 3a gives the XRD diagram of GO, MCNTs and MCG-5. It can be seen that a strong characteristic diffraction peak exists at $11.2^{\circ}(d=0.79 \mathrm{~nm})$ for $\mathrm{GO}$ and at $25.8^{\circ}(d=0.34 \mathrm{~nm})$ [35] for MCNTs. After the hydrothermal reaction, $\mathrm{GO}$ was reduced and some oxidation functional groups were reduced, leading to re-stacking of some graphene sheets. The MCNTs that overlapped on the graphene layer reduced the disordered layer stacking to a certain extent. So, MCG-5 has a weak peak at $25.6^{\circ}[36]$ in its XRD spectrum. The Raman spectrum was shown in Fig. $3 \mathrm{~b}$, $D$ peak and $G$ peak existing at $1351 \mathrm{~cm}^{-1}$ and $1587 \mathrm{~cm}^{-1}$ respectively. Both of MCG-5 and MCNTs have $2 D$ peak at $2691 \mathrm{~cm}^{-1}$. The $D$ peak is related to the defect position and the random property of the graphite structure. And the 2D peak is the characteristic peak of graphene [37]. The intensity ratio between $D$ peak and $G$ peak of GO, MCNTs and MCG-5 $\left(\mathrm{I}_{\mathrm{D}} / \mathrm{I}_{\mathrm{G}}\right)$ are $0.86,1.44$ and 1.27 respectively. This result indicates that defects formed on the graphene layers during the hydrothermal reduction process. Also, the MCNTs itself has more defect positions, which together with that on graphene layers form the defect position of MCG-5. The more the defect position it has, the stronger the adsorption capacity is. Therefore, the addition of MCNTs and the
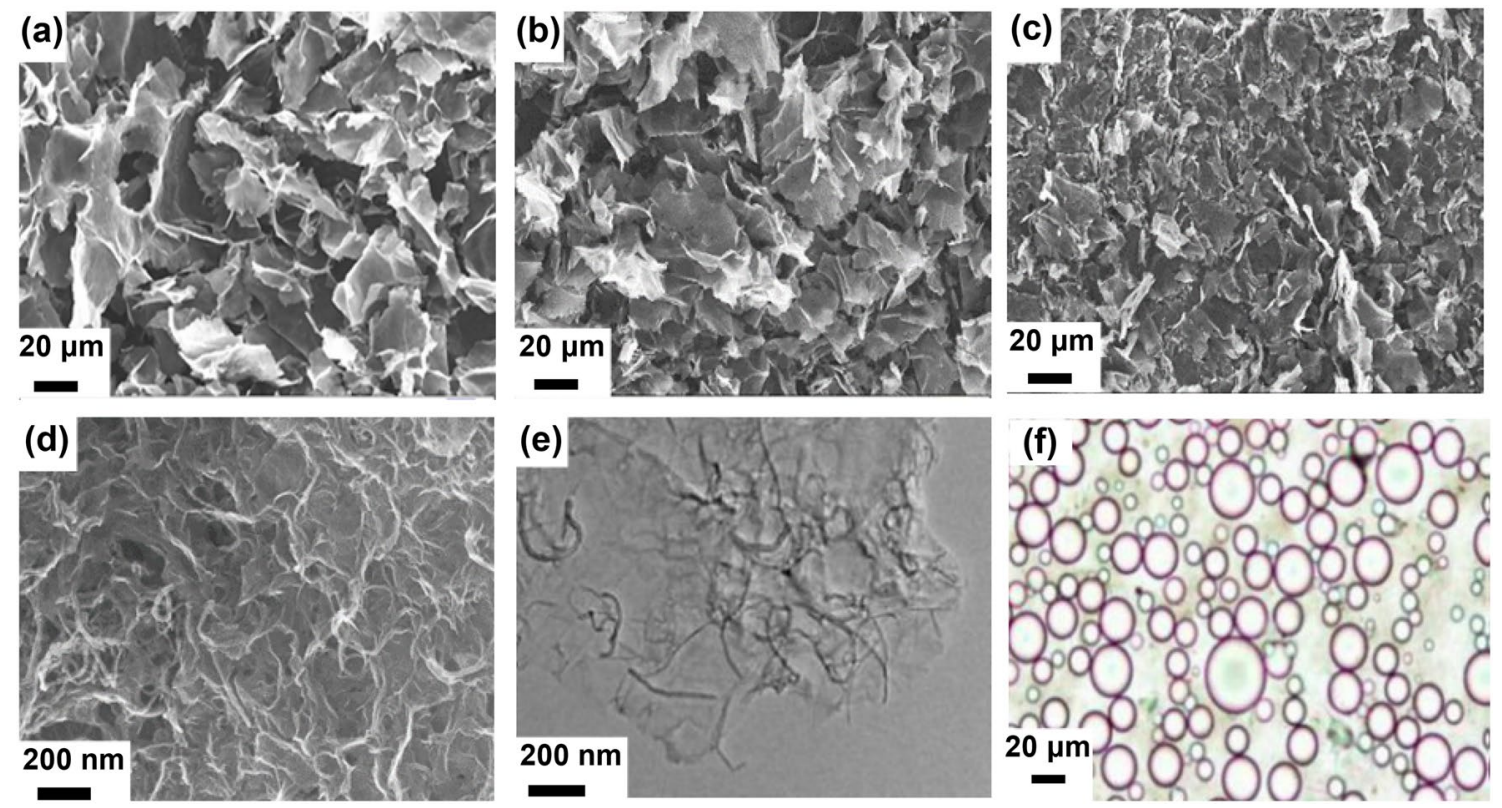

Fig. 2 SEM images at low-magnification of MCG-2, MCG-5 and MCG-8 (a-c); SEM images of MCG-5 at high-magnification (d); TEM images of MCG-5 (e); optical microscopy image of emulsion under T/S being 10:5 (f) 
Fig. 3 XRD spectra (a) and Raman spectra (b) of GO, MCNTs, MCG-5
Fig. 4 C $1 s$ narrow scan spectra (a) and XPS wide scan spectra (b) of MCG-5 (a)

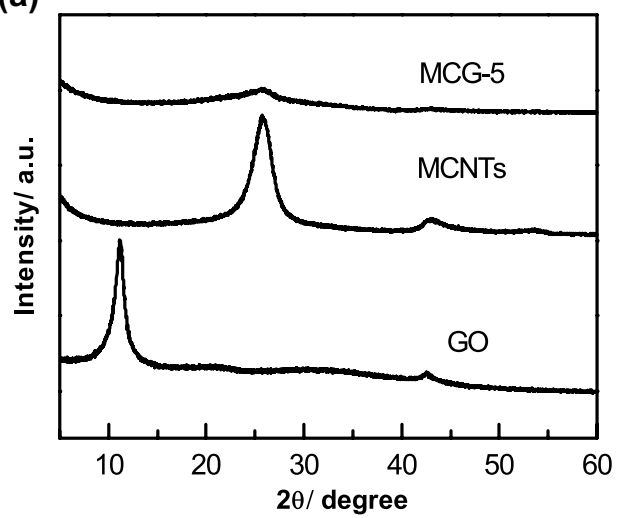

(a)

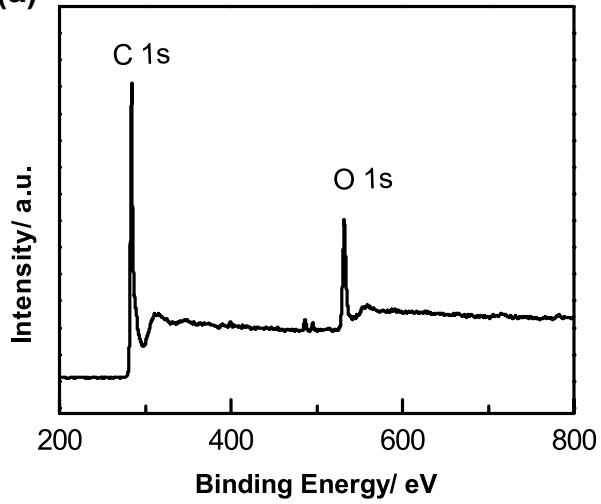

(b)

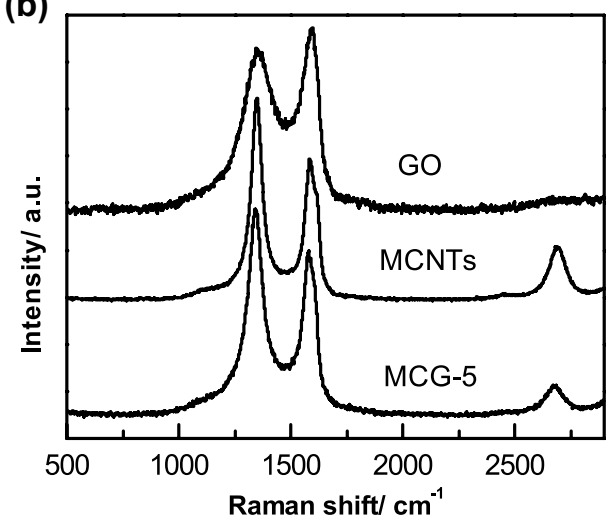

(b)

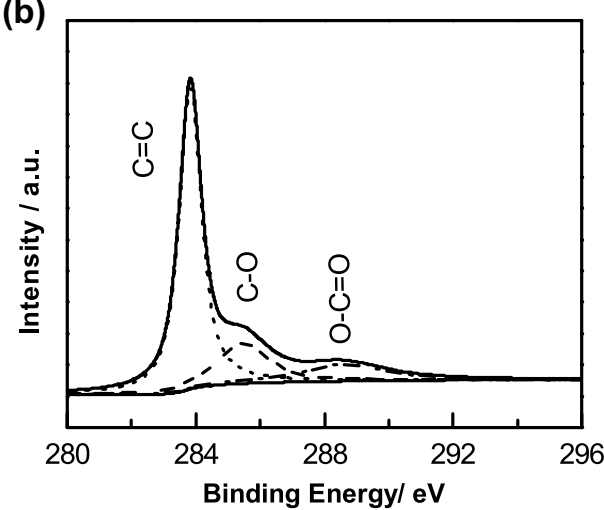

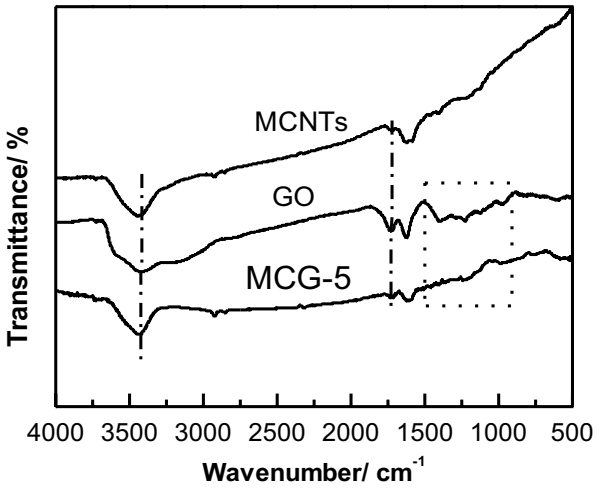

Fig. 5 FT-IR spectra of GO, MCNTs and MCG-5

hydrothermal reduction process can enhance the MCG-5 adsorption performance [38].

The chemical composition of MCG- 5 is measured by XPS. As shown in Fig. 4, the C/O atom ratio of MCG-5 is 5.96. And the $\mathrm{C} 1 \mathrm{~s}$ peak is composed of $\mathrm{C}=\mathrm{C} / \mathrm{C}-\mathrm{C}(283.8 \mathrm{eV}), \mathrm{C}-\mathrm{O}$ $(285.5 \mathrm{eV})$ and $\mathrm{O}-\mathrm{C}=\mathrm{O}(288.6 \mathrm{eV})$ peaks [34, 39]. Among them, $C=C / C-C$ accounted for 0.66 , indicating that most of the oxygen functional groups on $\mathrm{GO}$ and MCNTs were removed and $\mathrm{C}=\mathrm{O}$ functional groups were mostly reduced.
Figure 5 is a Fourier infrared spectra of MCNTs, GO and MCG-5. It can be seen that MCNTs has obvious $\mathrm{O}-\mathrm{C}=\mathrm{O}$ characteristic peaks at $1733 \mathrm{~cm}^{-1}$, indicating that $\mathrm{O}-\mathrm{C}=\mathrm{O}$ functional groups are generated after acidification. The following characteristic peaks appear in the $\mathrm{GO}$ spectrum [40]: $3432 \mathrm{~cm}^{-1}$ (O-H telescopic vibration), $1731 \mathrm{~cm}^{-1}(\mathrm{C}=\mathrm{O}$ telescopic vibration), $1623 \mathrm{~cm}^{-1}(\mathrm{C}=\mathrm{C}$ telescopic vibration), $1399 \mathrm{~cm}^{-1}$ (O-H deformed vibration) and $974 \mathrm{~cm}^{-1}$ (C-O-C). After hydrothermal reduction, most of the MCG-5 oxygen functional group $(\mathrm{C}-\mathrm{O}-\mathrm{C} / \mathrm{C}=\mathrm{O})$ were removed. But there are still distinct characteristic peaks at 3432 and $1731 \mathrm{~cm}^{-1}$, indicating that most of the $\mathrm{O}-\mathrm{C}=\mathrm{O}$ are retained in MCG-5, which is consistent with the XPS analysis.

\subsection{The effect of MCG types and temperature on the adsorption of MB}

The SEM characterization results show that MCG has porous structure and the pore sizes are in micron and nanoscale. Also, the characterization results of FT-IR and XPS indicate that MCG contains oxygen functional groups $(\mathrm{O}-\mathrm{C}=\mathrm{O} / \mathrm{C}-\mathrm{O}$ and so on) and $\pi-\pi$ conjugated bonds formed on graphene lamellar. So the internal surface of MCG provides a large number of adsorption sites, which is beneficial to the 
adsorption of MB molecules. It is found that the pore size of MCG changed with the changing of T/S ratio in the emulsion. $M B$ in water was adsorbed by different MCGs, as shown in Fig. 6a. At the first $400 \mathrm{~min}, \mathrm{MB}$ was adsorbed quickly on MCGs, then the increase of adsorption capacity slowed down and finally approached equilibrium. The removal rate of MB onto MCG-2, 5 and 8 were all more than $90 \%$. The MB solution after adsorption experiment became colorless and just like water. All of these results can indicate that MCGs have excellent adsorption property for MB. The equilibrium adsorption capacities of MCG-2, 5 and 8 are 164.1, 199.2 and $233.8 \mathrm{mg} \mathrm{g}^{-1}$, respectively. Because the pore size of MCG-8 is the smallest, so it has the maximum adsorption surface area [41]. The equilibrium adsorption capacities show that the adsorption of MCG on MB can be effectively changed by changing the preparation conditions.

The adsorption temperature is also an important factor. In the range of 298-328 K, the adsorption amount of MB on MCG- 5 varies with time, as shown in Fig. $6 \mathrm{~b}$. The equilibrium adsorption capacity of MCG-5 at $298 \mathrm{~K}$ is $199.2 \mathrm{mg} \mathrm{g}^{-1}$; While it increases to $253.1 \mathrm{mg} \mathrm{g}^{-1}$ at $328 \mathrm{~K}$, indicating that the higher the adsorption temperature is, the higher the adsorption capacity is.

\subsection{Adsorption equilibrium}

The adsorption isothermal model can be used to analyze the distribution of MB molecules in the two phase of solid and liquid during adsorption equilibrium. The adsorption isotherm model is usually related to the nature of adsorbed molecules [42]. There are two common models of isothermal adsorption: Langmuir model and Freundlich model.

The Langmuir model [43] assumes that the surface of the adsorbent is homogeneous and that each adsorption site can only be occupied by one adsorbed molecule. The mathematical expression is shown in Eq. (2).

$\frac{C_{e}}{q_{e}}=\frac{C_{e}}{q_{m}}+\frac{1}{K_{L} q_{m}}$ where $q_{e}$ and $q_{m}$ are the equilibrium adsorption capacity and the saturated adsorption capacity of MCG-5, $\mathrm{mg} \mathrm{g}^{-1}$; $K_{L}$ is adsorption equilibrium constant in Langmuir model, $\mathrm{L} \mathrm{mg}^{-1} ; C_{e}$ is the equilibrium concentration in the liquid phase, $\mathrm{mg} \mathrm{L}^{-1}$.

The Freundlich model [44] is an empirical equation. The basic assumption is that the adsorption occurs on the nonuniform surface. It is shown in Eq. (3).

$\ln q_{e}=\ln K_{F}+\frac{1}{n} \ln C e$

where $n$ and $K_{F}$ are the constants in Freundlich model. 1/n reflects whether the adsorption process is favorable and $K_{F}$ is the amount of MB molecules adsorbed by MCG- 5 under unit equilibrium concentration.

Figure 7 shows the fitting curves of $q_{e} \sim C_{e}$ for the two models. And data of the fitting results are shown in Table 1. The Decision coefficient, $R^{2}$ of Freundlich model is 0.9166 , the model constants $1 / n$ and $K_{F}$ are 0.2008 and 165.7 , respectively. Although $R^{2}$ is not high, the adsorption performance can be evaluated by the $1 / n$ value. Generally the adsorption is easy to occur when $1 / n$ is in the range of $0.1-1$. So, it is expected that adsorption of MB in water onto MCG-5 is easy to occur. $R^{2}$ of Langmuir model fitting is 0.9994 , which

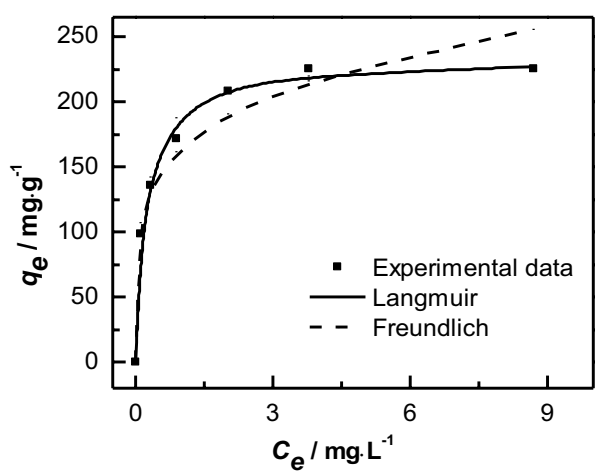

Fig. 7 Langmuir and Freundlich isotherms for MB onto MCG-5 at $298 \mathrm{~K}$
Fig. 6 Effect of MCG types (a) and adsorption temperature (MCG-5) (b) for MB adsorption

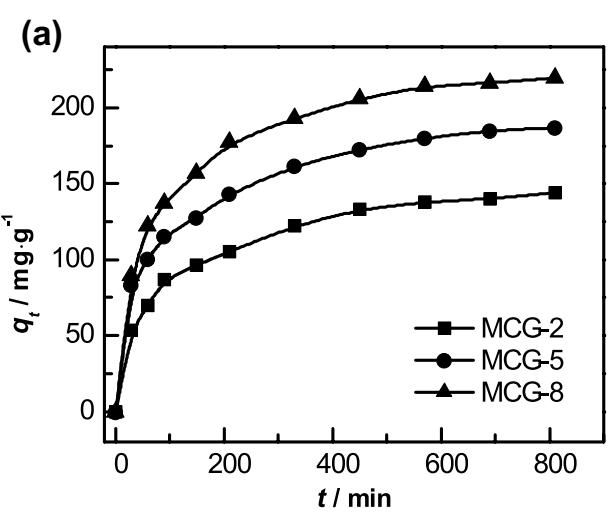

(b)

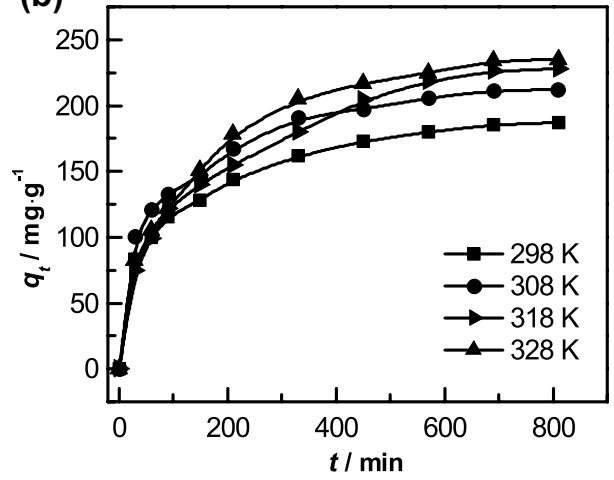


Table 1 Isotherm parameters of MB adsorbed onto MCG-5 at $298 \mathrm{~K}$

\begin{tabular}{|c|c|c|c|c|c|}
\hline \multicolumn{3}{|l|}{ Langmuir } & \multicolumn{3}{|c|}{ Freundlich } \\
\hline$K_{L}\left(\mathrm{~L} \mathrm{mg}^{-1}\right)$ & $q_{m}\left(\mathrm{mg} \mathrm{g}^{-1}\right)$ & $R^{2}$ & $K_{F}$ & $1 / n$ & $R^{2}$ \\
\hline 4.656 & 232 & 0.9994 & 165.7 & 0.2008 & 0.9166 \\
\hline
\end{tabular}

Table 2 Saturation adsorption capacities for MB onto various adsorbents

\begin{tabular}{lll}
\hline Adsorbent & $q_{m}\left(\mathrm{mg} \mathrm{g}^{-1}\right)$ & References \\
\hline MCG-5 & 232 & This work \\
ACPs-20 & 146.5 & {$[2]$} \\
GOs & $120.32-158.27$ & {$[3]$} \\
RGO-SA & 192.3 & {$[16]$} \\
M-MWCNTs & 48.06 & {$[23]$} \\
GF & $90.7-215.35$ & {$[40]$} \\
emGA & 166 & {$[45]$} \\
G-CNT & 81.97 & {$[46]$} \\
GNS/Fe ${ }_{3}$ H $_{4}$ & 43.82 & {$[47]$} \\
QDs-MSN & 73.15 & {$[48]$} \\
Active carbon & 104.3 & {$[49]$} \\
Chaff & 20.3 & {$[50]$} \\
Pine cone biomass & 109.89 & {$[51]$} \\
\hline
\end{tabular}

is obviously higher than that of Freundlich model, indicating that the adsorption process is monolayer adsorption. $K_{L}$ is $4.656 \mathrm{~L} \mathrm{mg}^{-1}$ and $q_{m}$ is $232 \mathrm{mg} \mathrm{g}^{-1}$. In order to compare the adsorption capacity of different adsorbents for $M B$, adsorption capacity of MCG-5 and other common adsorption agent are summarized in Table 2 . The results showed that the saturated adsorption capacity of MCG-5 is obviously higher than that of other natural adsorption materials, and also higher than that of other three dimensional porous carbon materials.

\subsection{Adsorption thermodynamics}

Thermodynamic parameters such as standard enthalpy change $\left(\Delta H^{0}\right)$, standard entropy change $\left(\Delta S^{0}\right)$ and standard Gibbs free energy change $\left(\Delta G^{0}\right)$, etc. can give information of adsorption mechanism. These data can be obtained from Eqs. (4) to (5) [52].

$$
\begin{aligned}
& \ln K_{d}=\frac{\Delta S^{0}}{R}-\frac{\Delta H^{0}}{R T} \\
& K_{d}=\frac{q_{e}}{C_{e}}
\end{aligned}
$$

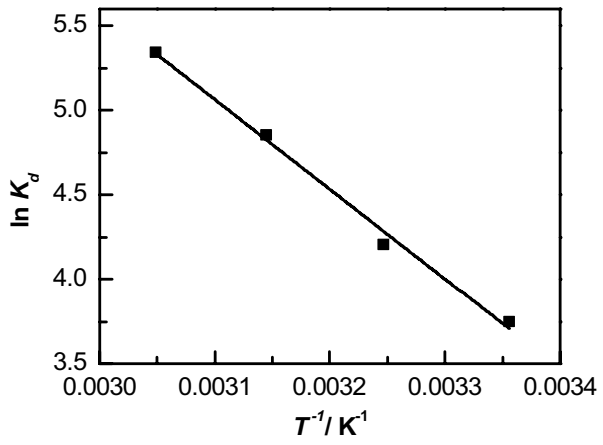

Fig. 8 The curve of $\ln K_{d} \sim T^{-1}$

Table 3 Thermodynamic parameters in the adsorption process of MB for MCG-5

\begin{tabular}{llllll}
\hline$\Delta G^{0}\left(\mathrm{~kJ} \mathrm{~mol}^{-1}\right)$ & & & $\Delta H^{0}\left(\mathrm{~kJ} \mathrm{~mol}^{-1}\right)$ & $\begin{array}{l}\Delta S^{0} \\
\left(\mathrm{~kJ} \mathrm{~mol}^{-1} \mathrm{~K}^{-1}\right)\end{array}$ \\
\cline { 1 - 5 } $298 \mathrm{~K}$ & $308 \mathrm{~K}$ & $318 \mathrm{~K}$ & $328 \mathrm{~K}$ & & \\
\hline-9.19 & -10.98 & -12.77 & -14.55 & 44.06 & 0.1787 \\
\hline
\end{tabular}

where $\Delta H^{0}$ is the standard enthalpy change, $\mathrm{kJ} \mathrm{mol}^{-1} ; \Delta S^{0}$ is the standard entropy change, $\mathrm{kJ} \mathrm{mol}^{-1} \mathrm{~K}^{-1} ; R$ is the gas constant, $\mathrm{J} \mathrm{mol}^{-1} \mathrm{~K}^{-1} ; T$ is the adsorption temperature, $\mathrm{K}$.

In addition, the $\Delta G^{0}$ can be calculated by Eq. (6) [52].

$\Delta G^{0}=\Delta H^{0}-\Delta S^{0} T$

The adsorption equilibrium data $\ln K_{d} \sim T^{-1}$ are plotted in Fig. 8. According to the slope and intercept of the fitting curve, the $\Delta H^{0}, \Delta S^{0}$ and $\Delta G^{0}$ can be calculated, and the results are summarized in Table 3. Both $\Delta H^{0}$ and $\Delta S^{0}$ are positive, indicating that the adsorption of $\mathrm{MB}$ by adsorbents is an endothermic process and the MB adsorption is an entropy increasing process, indicating the increasing affinity of MCG-5 for MB molecules [42,52]. The adsorption process with $\Delta G^{0}$ in the range of 0 to $-20 \mathrm{~kJ} \mathrm{~mol}^{-1}$ belongs to physical adsorption, while that in the range of -80 to $-400 \mathrm{~kJ} \mathrm{~mol}^{-1}$ belongs to chemisorption [53]. Data in Table 1 shows that when the temperature changed from 298 to $328 \mathrm{~K}, \Delta G^{0}$ changed from -9.19 to $-14.55 \mathrm{~kJ} \mathrm{~mol}^{-1}$, meaning the adsorption of MB on MCG- 5 is a physical absorption process, and the negative value indicates that the adsorption process is spontaneous.

\subsection{Adsorption kinetics}

The pseudo-first-order kinetic model [54] and the pseudosecond-order kinetic model [55] are commonly used to study the mechanism and adsorption rate of the adsorption process. The mathematical expressions of the two models are shown as Eqs. (7) and (8).

$\ln \left(q_{e}-q_{t}\right)=\ln q_{e}-k_{1} t$ 
Fig. 9 Pseudo-first-order (a) and pseudo-second-order (b) kinetics plots of $\mathrm{MB}$ adsorption onto MCG at $298 \mathrm{~K}$

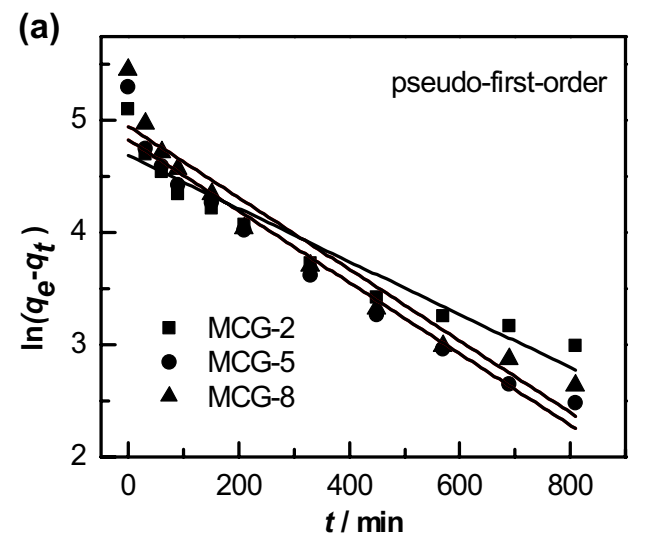

Table 4 Parameters of pseudo first- and second-order kinetics for MB on MCG at $298 \mathrm{~K}$

\begin{tabular}{|c|c|c|c|c|c|c|c|c|c|}
\hline \multirow[t]{2}{*}{ MCG } & \multirow[t]{2}{*}{$q_{e, \text { exp }}\left(\mathrm{mg} \mathrm{g}^{-1}\right)$} & \multicolumn{4}{|c|}{ Pseudo-first-order } & \multicolumn{4}{|c|}{ Pseudo-second-order } \\
\hline & & $\overline{q_{e, \text { cal }}\left(\mathrm{mg} \mathrm{g}^{-1}\right)}$ & $k_{1}\left(\min ^{-1}\right)$ & $R^{2}$ & $\overline{S D}(\%)$ & $\overline{q_{e, c a l}\left(\mathrm{mg} \mathrm{g}^{-1}\right)}$ & $k_{2}\left(\mathrm{~g} \mathrm{mg}^{-1} \mathrm{~min}^{-1}\right)$ & $R^{2}$ & SD (\%) \\
\hline MCG-2 & 164.1 & 108.5 & 0.00236 & 0.9171 & 55.42 & 156.7 & $7.93 \times 10^{-5}$ & 0.9975 & 6.86 \\
\hline MCG-5 & 199.2 & 125.0 & 0.00318 & 0.9530 & 54.40 & 201.6 & $7.21 \times 10^{-5}$ & 0.9974 & 8.45 \\
\hline MCG-8 & 233.8 & 141.1 & 0.00319 & 0.9321 & 56.08 & 235.8 & $6.67 \times 10^{-5}$ & 0.9990 & 5.17 \\
\hline
\end{tabular}

$\frac{t}{q_{t}}=\frac{1}{k_{2} q_{e}^{2}}+\frac{t}{q_{e}}$

where $k_{1}$ is the pseudo-first-order kinetic adsorption rate constant, $\min ^{-1} ; k_{2}$ is the pseudo-second-order kinetic adsorption rate constant, $\mathrm{g} \mathrm{mg}^{-1} \mathrm{~min}^{-1} ; q_{e}$ and $q_{t}$ are the capacity at equilibrium state and that at $t$ moments respectively, $\mathrm{mg} \mathrm{g}^{-1}$.

The kinetics data of MCG-2, 5, 8 are fitted using Eqs. (7) and (8), as shown in Fig. 9. It can be seen from the diagram that the fitting effect of pseudo-first-order kinetic equation is poor, with $R^{2}$ being $0.9171-0.9530$, and the standard deviation is higher than 54\%. The fitted results of pseudo-second-order kinetic equation coincide with the actual adsorption data perfectly, with $R^{2}$ being greater than 99\%, and the standard deviation is lower than 9\%. MCG-5 is used as an example to calculate the equilibrium adsorption capacity, which is $201.6 \mathrm{mg} \mathrm{g}^{-1}$, in agreement with the experimental value $199.2 \mathrm{mg} \mathrm{g}^{-1}$. The fitting results are shown in Table 4 . The adsorption rate constant $k_{2}$ is $7.93 \times 10^{-5}$ and $6.67 \times 10^{-5} \mathrm{~g} \mathrm{mg}^{-1} \mathrm{~min}^{-1}$ for MCG-2 and MCG-8, respectively. The SEM characterization results have shown that the pore size of MCG-2 is the largest while MCG-8 is the smallest. It can be seen that the adsorption rate decreases with the decrease of the pore size. It is expected that the MB solution is easier to enter into pores with larger size, leading to higher adsorption rate [2].

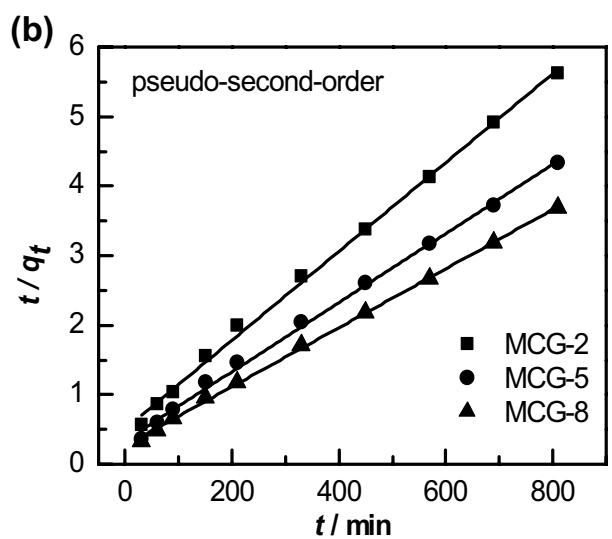


good adsorbent for MB for practical application. Also, the further industrial mass production of MCG can be considered in the future.

\subsection{Adsorption mechanism of methylene blue and Challenges}

Because there are many factors that can influence the adsorption process. The adsorption mechanism of methylene blue has been reported widely in many related references. Zhao et al. [56] reported that MB molecules can transfer from solution to the surface of catalyst and be adsorbed with offset face-to-face orientation via $\pi-\pi$ conjugation between $\mathrm{MB}$ and aromatic regions of the graphene. In the paper of Ma et al. [16], it was found that the adsorption mechanism of MB was different on two different adsorbents. The negative charge of oxygen containing functional groups on the GO-SA surface were favorable for the adsorption of cationic MB. RGO-SA had aromatic ring structure, and $M B$ molecule produced $\pi-\pi$ conjugation with RGO-SA. Also, Zheng et al. [57] found that the main mechanism of $\mathrm{Pb}$ (II) adsorption on $\beta-C D-G O$ is surface complexation and electrostatic interaction. And $\pi-\pi$ interaction is the main adsorption mechanism of 1-naphthol on $\beta$-CD-GO. So, we can know that adsorption is a complex process after all. There are different adsorption mechanism when different adsorbents adsorb different substances. Therefore, not only can it be influenced by the $\pi-\pi$ conjugation between MB and aromatic regions of the graphene, but also it is related to the physicochemical properties of carbon-based adsorbents.

In a word, it is difficult to come to the same conclusions due to inconsistent experimental conditions. Therefore, it is necessary to study the effects of the various influencing factors under a uniform experimental condition. Meanwhile, there are huge differences in the chemical composition of natural surface water. There is still a great knowledge gap between the simplified laboratory results and the actual behavior of carbon materials in natural water. We should do more further investigation about this work in the future.

\section{Conclusions}

1. GO was used as emulsion stabilizer, and homogeneous emulsion was obtained after high-speed stirring. Then MCG was prepared through hydrothermal reaction. It was observed that MCG had rich micro-pores from SEM and TEM images. When the T/S ratio increased, the size of the pore size decreased. The MCNTs interlaced on the graphene layer, which enhanced the wrinkle of the graphene lamellar and the nanoscale pores. the
XPS and FT-IR analysis shows that the $\mathrm{C} / \mathrm{O}$ atom ratio of MCG was 5.96 and the oxygen containing functional group was $\mathrm{O}-\mathrm{C}=\mathrm{O} / \mathrm{C}-\mathrm{O}$.

2. The equilibrium adsorption capacity of MCG-2, MCG-5 and MCG-8 is $164.1,199.2$ and $233.8 \mathrm{mg} \mathrm{g}^{-1}$, respectively. The smaller the pore size is, the greater the equilibrium adsorption is. The adsorption thermodynamic parameters such as $\Delta H^{0}, \Delta S^{0}$ and $\Delta G^{0}$ show that the adsorption process of MB on MCG is physical adsorption, and the higher the temperature is, the better the adsorption effect is. The adsorption isotherm of MCG-5 is better fitted by Langmuir model. The saturated adsorption capacity is $232 \mathrm{mg} \mathrm{g}^{-1}$, which is higher than that of common adsorbent.

3. The adsorption process of MB onto different kinds of MCG conforms to the pseudo-second-order kinetics model. The adsorption rate is related to the pore size, and the smaller the pore size is, the slower the adsorption rate is.

4. Adsorption mechanism of methylene blue is complex. Not only can it be influenced by the $\pi-\pi$ conjugation between MB and aromatic regions of the graphene, but also it is related to the physicochemical properties of carbon-based adsorbents.

5. The results of cyclic adsorption showed that MCG has good property that can still maintain a high adsorption capacity after 5 cycles. So, the MCG can be used as a kind of excellent adsorbent for MB dye and other sewage and waste-water polluted by MB. Also, the further industrial mass production of MCG can be considered in the future.

Acknowledgements All the authors of this article thank for the support of the Natural Science Foundation of Shandong Province (ZR2017MB015), Projects of State Key Laboratory of Heavy Oil Processing (SLKZZ-2017002), PetroChina Innovation Foundation (2017D-5007-0601) and the Postgraduate Innovation Project of China University of Petroleum (East China) (YCX2017036).

Funding This study was funded by the Natural Science Foundation of Shandong Province (ZR2017MB015), Projects of State Key Laboratory of Heavy Oil Processing (SLKZZ-2017002), PetroChina Innovation Foundation (2017D-5007-0601) and the Postgraduate Innovation Project of China University of Petroleum (East China) (YCX2017036).

\section{Compliance with ethical standards}

Conflict of interest The authors declare that they have no conflict of interest. 


\section{References}

1. Bao N, Li Y, Wei Z, Yin G, Niu J (2011) Adsorption of dyes on hierarchical mesoporous $\mathrm{TiO}_{2}$ fibers and its enhanced photocatalytic properties. J Phys Chem C 115(13):5708-5719

2. Suo L, Shengjuan LI, Yingtao LI, Li Z, Xi Z (2016) Kinetic studies of all-carbon porous structure on the adsorption of methylene blue. Chem J Chin Univ 37(11):2043-2049

3. Yong T, Zhong G, Wang X, Lin H (2012) Preparation of high specific surface area magnetic ordered mesoporous carbons and adsorption property of methylene blue. CIESC J 63:4082-4088

4. Wei G, Miao Y, Zhang C, Yang Z, Liu Z, Weng WT et al (2013) $\mathrm{Ni}$-doped graphene/carbon cryogels and their applications as versatile sorbents for water purification. Appl Mater Interfaces 5(15):7584

5. Wang C, Shi ZH, Peng L, He WM, Li BL, Li KZ (2017) Preparation of carbon foam-loaded nano- $\mathrm{TiO}_{2}$, photocatalyst and its degradation on methyl orange. Surf Interfaces 7:116-124

6. Khalid NR, Ahmed E, Hong Z, Zhang Y, Ullah M, Ahmed M (2013) Graphene modified Nd/TiO , photocatalyst for methyl orange degradation under visible light irradiation. Ceram Int 39(4):3569-3575

7. Kim TH, Park C, Yang J, Kim S (2004) Comparison of disperse and reactive dye removals by chemical coagulation and fenton oxidation. J Hazard Mater 112(1-2):95

8. He Y, Li G, Wang H, Jiang Z, Zhao J, Su H et al (2010) Diafiltration and water recovery of reactive brilliant blue $\mathrm{KN}-\mathrm{R}$ solution by two-stage membrane separation process. Chem Eng Process 49(5):476-483

9. Ai L, Jiang J (2012) Removal of methylene blue from aqueous solution with self-assembled cylindrical graphene-carbon nanotube hybrid. Chem Eng J 192(2):156-163

10. Wu Y, Zhang L, Gao C, Ma J, Ma X, Han R (2009) Adsorption of copper ions and methylene blue in a single and binary system on wheat straw. J Chem Eng Data 54(54):3229-3234

11. Hameed BH, Din AT, Ahmad AL (2007) Adsorption of methylene blue onto bamboo-based activated carbon: kinetics and equilibrium studies. J Hazard Mater 141(3):819-825

12. Parida KM, Sahu S, Reddy KH, Sahoo PC (2013) A kinetic, thermodynamic, and mechanistic approach toward adsorption of methylene blue over water-washed manganese nodule leached residues. Ind Eng Chem Res 50(2):843-848

13. Zhu Y, Murali S, Cai W, Li X, Suk JW, Potts JR et al (2010) Graphene and graphene oxide: synthesis, properties, and applications. Adv Mater 22(35):3906-3924

14. Loh KP, Bao Q, Ang PK, Yang J (2010) The chemistry of graphene. J Mater Chem 20(12):2277-2289

15. Lee C, Wei X, Kysar JW, Hone J (2008) Measurement of the elastic properties and intrinsic strength of monolayer graphene. Science 321(5887):385-388

16. Ma T, Chang PR, Zheng P, Zhao F, Ma X (2014) Fabrication of ultra-light graphene-based gels and their adsorption of methylene blue. Chem Eng J 240(6):595-600

17. Ji L, Chen W, Duan L, Zhu D (2009) Mechanisms for strong adsorption of tetracycline to carbon nanotubes: a comparative study using activated carbon and graphite as adsorbents. Environ Sci Technol 43(7):2322-2327

18. Ai L, Zhang C, Liao F, Wang Y, Li M, Meng L et al (2011) Removal of methylene blue from aqueous solution with magnetite loaded multi-wall carbon nanotube: kinetic, isotherm and mechanism analysis. J Hazard Mater 198(2):282-290

19. Baughman RH, Zakhidov AA, Heer WAD (2002) Carbon nanotubes - the route toward applications. Science 297(5582):787-792
20. Ichihashi T, lijima S (1993) Single-shell carbon nanotubules of 1-nm diameter. Nat Int Wkly J Sci 363:603-605

21. Pan ZW, Wang ZL (2001) Nanobelts of semiconducting oxides. Science 291(5510):1947-1949

22. Zhao Z, Xu B, Wang LM, Zhou XF, He J, Liu Z et al (2011) Three dimensional carbon-nanotube polymers. ACS Nano 5(9):7226-7234

23. Falvo MR, Clary GJ, Taylor li RM, Chi V, Brooks FP Jr et al (1997) Bending and buckling of carbon nanotubes under large strain. Nature 389(6651):582

24. Sun H, Xu Z, Gao C (2013) Multifunctional, ultra-flyweight, synergistically assembled carbon aerogels. Adv Mater 25(18):2554-2560

25. Liu YF, Yuan GH, Jiang ZH, Yao ZP, Yue M, Liu YF et al (2015) Preparation of $\mathrm{Ni}(\mathrm{OH}) 2$-graphene sheet-carbon nanotube composite as electrode material for supercapacitors. J Alloys Compd 618:37-43

26. Wan W, Zhang R, Li W, Liu H, Lin Y, Li L et al (2016) Graphenecarbon nanotube aerogel as an ultra-light, compressible and recyclable highly efficient absorbent for oil and dyes. Environ Sci Nano 3(1):107-113

27. Huang X, Qian K, Yang J, Zhang J, Li L, Yu C et al (2012) Functional nanoporous graphene foams with controlled pore sizes. Adv Mater 24(32):4419-4423

28. Kim J, Cote LJ, Kim F, Yuan W, Shull KR, Huang J (2010) Graphene oxide sheets at interfaces. J Am Chem Soc 132(23):8180

29. Hummers WS Jr, Offeman RE (1958) Preparation of graphitic oxide. J Am Chem Soc 80(6):1339

30. Cote LJ, Kim F, Huang J (2009) Langmuir-blodgett assembly of graphite oxide single layers. J Am Chem Soc 131(3):1043-1049

31. Liang D, Gao J, Sun H, Chen P, Hou Z, Zheng X (2011) Selective oxidation of glycerol with oxygen in a base-free aqueous solution over MWNTs supported Pt catalysts. Appl Catal B 106(3):423-432

32. He Y, Wu F, Sun X, Li R, Guo Y, Li C et al (2013) Factors that affect pickering emulsions stabilized by graphene oxide. Appl Mater Interfaces 5(11):4843-4855

33. Zhao J, Ren W, Cheng HM (2012) Graphene sponge for efficient and repeatable adsorption and desorption of water contaminations. J Mater Chem 22(38):20197-20202

34. Shen Y, Zhu X, Zhu L, Chen B (2017) Synergistic effects of 2D graphene oxide nanosheets and 1D carbon nanotubes in the constructed 3D carbon aerogel for high performance pollutant removal. Chem Eng J 314:336-346

35. Kabiri S, Tran DNH, Altalhi T, Losic D (2014) Outstanding adsorption performance of graphene-carbon nanotube aerogels for continuous oil removal. Carbon 80(1):523-533

36. Li J, Li J, Meng H, Xie S, Zhang B, Li L et al (2014) Ultra-light, compressible and fire-resistant graphene aerogel as a highly efficient and recyclable absorbent for organic liquids. J Mater Chem A 2(9):2934-2941

37. Hu H, Zhao Z, Gogotsi Y, Qiu J (2014) Compressible carbon nanotube-graphene hybrid aerogels with superhydrophobicity and superoleophilicity for oil sorption. Environ Sci Technol Lett 1(3):214-220

38. Zhang ZY, Xu XC (2015) Nondestructive covalent functionalization of carbon nanotubes by selective oxidation of the original defects with $\mathrm{K}_{2} \mathrm{FeO}_{4}$. Appl Surf Sci 346:520-527

39. Hu HB, Zhang T, Cui Z et al (2016) Preparation of three-dimensional honeycomb-like material of grapheme oxide-carboxylated carbon nanotube-polyethylenepolyamine to adsorb $\mathrm{CO}_{2}$. Chem Ind Eng Prog 35(11):3576-3584

40. Chen J, Chi F, Huang L, Zhang M, Yao B, Li Y et al (2016) Synthesis of graphene oxide sheets with controlled sizes from sieved graphite flakes. Carbon 110:34-40 
41. Deng W, Fang Q, Zhou X, Cao H, Liu Z (2016) Hydrothermal selfassembly of graphene foams with controllable pore size. RSC Adv 6(25):20843-20849

42. Li Y, Du Q, Liu T, Sun J, Wang Y, Wu S et al (2013) Methylene blue adsorption on graphene oxide/calcium alginate composites. Carbohydr Polym 95(1):501-507

43. Langmuir I (2015) The adsorption of gases on plane surfaces of glass, mica and platinum. J Chem Phys 40(12):1361-1403

44. Freundlich HMF (1906) Over the adsorption in solution. Z Phys Chem 57:385-471

45. Huang Y, Liu H, Ma YB et al (2018) Preparation of graphene aerogel by emulsion method and its chemical progress in adsorption of methylene blue in water. Chem Ind Eng Prog 8:3092-3099

46. Ai L, Jiang J (2012) Removal of methylene blue from aqueous solution with self-assembled cylindrical graphene-carbon nanotube hybrid. Chem Eng J 192(2):156-163

47. Ai L, Zhang C, Chen Z (2011) Removal of methylene blue from aqueous solution by a solvothermal-synthesized graphene/ magnetite composite. J Hazard Mater 192(3):1515-1524

48. Ferenczi S, Dévényi T (2015) $\mathrm{SnO}_{2}$ quantum dots decorated silica nanoparticles for fast removal of cationic dye (methylene blue) from wastewater. Chem Eng J 281 (4):482-490

49. Dhodapkar R, Rao NN, Pande SP, Kaul SN (2006) Removal of basic dyes from aqueous medium using a novel polymer: Jalshakti. Biores Technol 97(7):877

50. Han R, Wang Y, Pan H, Jie S, Jian Y, Lu Y (2006) Removal of methylene blue from aqueous solution by chaff in batch mode. $J$ Hazard Mater 137(1):550-557
51. Sen TK, Afroze S, Ang HM (2011) Equilibrium, kinetics and mechanism of removal of methylene blue from aqueous solution by adsorption onto pine cone biomass of pinus radiata. Water Air Soil Pollut 218(1-4):499-515

52. Wang P, Cao M, Wang C, Ao Y, Hou J, Jin Q (2014) Kinetics and thermodynamics of adsorption of methylene blue by a magnetic graphene-carbon nanotube composite. Appl Surf Sci 290(1):116-124

53. Wang QJ, Sun HJ, Peng TJ et al (2017) Influence of oxidation degree of grapheme oxide on adsorption performance for methylene blue. CIESC J 68(4):1712-1720

54. Chong C, Jie D, Bei L, Ai H, Xiang Z, Lang M et al (2013) Toward 3D graphene oxide gels based adsorbents for high-efficient water treatment via the promotion of biopolymers. J Hazard Mater 263(Pt 2):467-478

55. Ho YS, Mckay G (1999) Pseudo-second order model for sorption processes. Process Biochem 34(5):451-465

56. Zhao D, Sheng G, Chen C, Wang X (2012) Enhanced photocatalytic degradation of methylene blue under visible irradiation on graphene@ $\mathrm{TiO}_{2}$, dyade structure. Appl Catal B 111(2):303-308

57. Zheng $\mathrm{H}$, Gao $Y$, Zhu K, Wang $Q$, Wakeel $M$, Wahid $A$ et al (2018) Investigation of the adsorption mechanisms of $\mathrm{Pb}$ (II) and 1-naphthol by $\beta$-cyclodextrin modified graphene oxide nanosheets from aqueous solution. J Colloid Interface Sci 530:154-162 